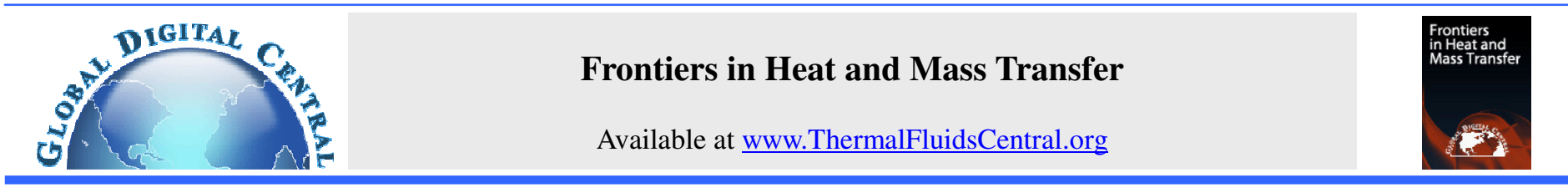

\title{
HEAT FLOW IN THIN FILMS VIA SURFACE PHONON-POLARITONS
}

\author{
Dye-Zone A. Chen and Gang Chen* \\ Department of Mechanical Engineering, Massachusetts Institute of Technology, Cambridge, MA, 02139, USA
}

\begin{abstract}
We present a calculation of the thermally generated electromagnetic flux propagating along the in-plane direction of a polar, thin film. The approach is based on fluctuational electrodynamics and the fluctuation-dissipation theorem. We find that for silicon carbide films between $5 \mathrm{~nm}$ and $100 \mathrm{~nm}$ thick, the thinner films transport more in-plane flux due to the long propagation length of the anti-symmetric surface phonon-polariton mode. Comparison of results obtained from the fluctuation-dissipation approach and the kinetic theory approach shows favorable agreement.
\end{abstract}

Keywords: heat conduction, phonon-polaritons, surface waves, thermal radiation, thin films

\section{INTRODUCTION}

Thermal conductivity is generally observed to decrease in structures with small length scales due to increased scattering of the heat carriers by interfaces and boundaries (Cahill et al., 2003; Goodson, 1996; Chen, 2001). These classical size effects become important when the characteristic dimension of the structure is smaller than the phonon mean free path. Recovery of this reduction in thermal conductivity is of interest in optoelectronics and microprocessors where heat dissipation is becoming one of the limiting factors in performance. Surface polaritons may provide a channel for this recovery.

Surface polaritons are hybrid electromagnetic waves that are the result of photons coupling to an elementary particle or excitation, such as an electron, phonon, or magnon. It is well-known that surface polaritons have long propagation lengths (Mills, 1975; Schoenwald et al., 1973), particularly on thin films (Burke et al., 1986), which in turn can lead to large in-plane energy flux (Chen et al., 2005), and also to increased heat transfer in out-of-plane near-field radiation (Carminati and Greffet, 1999; Mulet et al., 2001; Narayanaswamy and Chen, 2003; Shen et al., 2009). Additionally, in nanostructures surface effects are more important than volumetric effects due to a high surface area to volume ratio. This also suggests that surface polaritons may play an important role in energy transport along films with nanoscale thickness.

We present a calculation of the thermal energy flux propagating along the in-plane direction of a thin film via surface phononpolaritons. Our approach is based on fluctuational electrodynamics, which makes use of the fluctuation-dissipation theorem and dyadic Green's functions. We find that for silicon carbide films between $5 \mathrm{~nm}$ and $100 \mathrm{~nm}$ thick, the thinner films transport more in-plane flux due to the long propagation length of the anti-symmetric surface phononpolariton mode.

\section{FLUCTUATIONAL ELECTRODYNAMICS}

The fluctuational electrodynamics approach for calculating thermal radiation was pioneered by Rytov in 1958 (Rytov et al., 1989). This method includes the contributions due to evanescent waves which are not included in geometrical optics, and has been used to directly compute near-field thermal radiation and emission (Narayanaswamy and Chen, 2003; Narayanaswamy and Chen, 2005; Joulain et al., 2005). The fundamental idea is that the electric charges in a material in thermal equilibrium will experience random, thermal vibrations. These oscillating charges form fluctuating currents which are a source for electromagnetic radiation. The connection between the fluctuating current density and the temperature is given by the fluctuationdissipation theorem, which is discussed further below.

The physical system under consideration is shown in Fig. 1. The film is infinite in the $\hat{x}$ - and $\hat{y}$-directions, and has a thickness $d$ in the $\hat{z}$-direction. The material is polar and has a complex, frequency

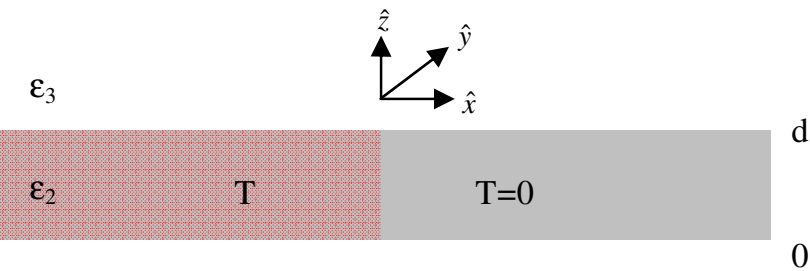

$\varepsilon_{1}$

Fig. 1 A thin film of polar material with thickness $d$ and dielectric function $\varepsilon_{2}(\omega)$ is surrounded on both sides by a vacuum with dielectric function $\varepsilon_{0}$. The film is assumed infinite and uniform in the $\hat{y}$-direction. In the $\hat{x}$-direction, from negative infinity to zero, the film is at temperature T. From zero to infinity, the film is assumed to be at $0 \mathrm{~K}$.

\footnotetext{
*Corresponding author: gchen2@mit.edu
} 
dependent dielectric function given by $\varepsilon_{2}(\omega)$ where $\omega$ is the angular frequency. The material is assumed to be isotropic, non-magnetic and is surrounded on either side by vacuum. To compute the in-plane flux, half of the film (from $x=-\infty$ to 0 ) is taken to be the source, and is assumed to be at a temperature $T$. The other half of the film is required to solve the electromagnetic problem, but is assumed to be at $0 \mathrm{~K}$. The temperature of the left-half of the film is uniform in the $\hat{y}$-direction, and the film is assumed thin enough that temperature variations in the $\hat{z}$ - direction are negligible.

\section{GREEN'S FUNCTIONS}

The electric and magnetic fields are obtained using the dyadic Green's functions (Tsang et al., 2000) of the vector Helmholtz equation, which are the spatial analogues to the impulse response or transfer function. In other words, they are used to calculate the electric and magnetic fields due to a point current source. For a source located at $\mathbf{r}^{\prime}$, the Fourier transform of the electric and magnetic fields at $\mathbf{r}$ are given by,

$$
\begin{aligned}
& \mathbf{E}(\mathbf{r}, \omega)=i \omega \mu_{o} \int_{V^{\prime}} d^{3} r^{\prime} \overrightarrow{\mathbf{G}}_{\mathbf{E}}\left(\mathbf{r}, \mathbf{r}^{\prime}, \omega\right) \cdot \mathbf{J}\left(\mathbf{r}^{\prime}, \omega\right) \\
& \mathbf{H}(\mathbf{r}, \omega)=\int_{V^{\prime}} d^{3} r^{\prime} \overrightarrow{\mathbf{G}}_{\mathbf{H}}\left(\mathbf{r}, \mathbf{r}^{\prime}, \omega\right) \cdot \mathbf{J}\left(\mathbf{r}^{\prime}, \omega\right)
\end{aligned}
$$

where $i$ is equal to $\sqrt{-1}, \mu_{0}$ is the permeability of free space, $\overrightarrow{\mathbf{G}}_{\mathbf{E}}\left(\mathbf{r}, \mathbf{r}^{\prime}, \omega\right)$ and $\overrightarrow{\mathbf{G}}_{\mathbf{H}}\left(\mathbf{r}, \mathbf{r}^{\prime}, \omega\right)$ are the electric and magnetic dyadic Green's functions which are related by $\overrightarrow{\mathbf{G}}_{\mathbf{H}}\left(\mathbf{r}, \mathbf{r}^{\prime}, \omega\right)=\nabla \times \overrightarrow{\mathbf{G}}_{\mathbf{E}}\left(\mathbf{r}, \mathbf{r}^{\prime}, \omega\right)$, $\mathbf{J}\left(\mathbf{r}^{\prime}, \omega\right)$ is the current density, and the integration is performed over $V^{\prime}$, which is the volume containing the source.

To calculate the power flow, we are interested in terms of the form $\left\langle E_{i}(\mathbf{r}, \omega) H_{j}^{*}(\mathbf{r}, \omega)\right\rangle$ where the brackets indicate the statistical ensemble average since the thermal radiation is stochastic, $i$ and $j$ denote the Cartesian coordinates, and the * indicates the complex conjugate. Using Eq. (1),

$$
\begin{aligned}
& \left\langle E_{i}(\mathbf{r}, \omega) H_{j}^{*}(\mathbf{r}, \omega)\right\rangle= \\
& i \omega \mu_{o} \int_{V^{\prime}} d^{3} r^{\prime} \int_{V^{\prime \prime}} d^{3} r^{\prime \prime} G_{E_{i l}}\left(\mathbf{r}, \mathbf{r}^{\prime}, \omega\right) G_{H_{j n}}^{T^{*}}\left(\mathbf{r}, \mathbf{r}^{\prime \prime}, \omega\right)\left\langle J_{l}\left(\mathbf{r}^{\prime}, \omega\right) J_{m}\left(\mathbf{r}^{\prime \prime}, \omega\right)\right\rangle
\end{aligned}
$$

where the superscript " $T$ " indicates the matrix transpose.

The Green's function used in these calculations was developed in terms of $s$ - and $p$-polarized waves which allows for the explicit inclusion of the Fresnel coefficients of a multilayer geometry (Joulain et al., 2005; Sipe, 1987). When the observation and source points are in the same layer, the electric Green's function is given by (Sipe, 1987),

$$
\begin{aligned}
& \overrightarrow{\mathbf{G}}_{\mathbf{E}}\left(\mathbf{r}, \mathbf{r}^{\prime}, \omega\right)= \\
& \int_{-\infty}^{\infty} \int_{-\infty}^{\infty} \frac{d k_{x} d k_{y}}{4 \pi^{2}} e^{i\left(k_{x}\left(x-x^{\prime}\right)+k_{y}(y-y)\right)}\left[\begin{array}{l}
\frac{i}{2 k_{z 2}}[\hat{s} \hat{s}+\hat{p} \hat{p}] e^{i k_{z 2} \mid z-z^{\prime}}-\frac{1}{k_{o}^{2} \varepsilon_{2}} \delta\left(z-z^{\prime}\right) \hat{z} \hat{z} \\
+\frac{i}{2 k_{z 2}}\left[\hat{s} \rho_{23}^{s} \hat{s}+\hat{p}_{2}^{+} \rho_{23}^{p} \hat{p}_{2}^{-}\right] e^{i k_{z 2}\left(z+z^{\prime}\right)} \\
+\frac{i}{2 k_{z 2}}\left[\hat{s} \rho_{21}^{s} \hat{s}+\hat{p}_{2}^{-} \rho_{21}^{p} \hat{p}_{2}^{+}\right] e^{i k_{z 2}\left(d-z+d-z^{\prime}\right)}
\end{array}\right]
\end{aligned}
$$

where

$$
\begin{aligned}
& k_{o}=\omega / c, k_{\rho}=\sqrt{k_{x}^{2}+k_{y}^{2}}, k_{z 2}=\sqrt{\varepsilon_{2} k_{o}^{2}-k_{x}^{2}-k_{y}^{2}}, \\
& \hat{s}=\left(k_{y} \hat{x}-k_{x} \hat{y}\right) / k_{\rho}, \hat{p}=\left(k_{\rho} / k_{2}-k_{z 2}\left(z-z^{\prime}\right) /\left(\left|z-z^{\prime}\right| k_{2}\right)\right) \hat{z},
\end{aligned}
$$

$\hat{p}_{2}^{ \pm}=-k_{z 2}\left(k_{x} \hat{x}+k_{y} \hat{y}\right) /\left(k_{2} k_{\rho}\right) \pm k_{\rho} / k_{2} \hat{z}$,

$\rho_{2 j}^{s, p}=r_{2 j}^{s, p} /\left(1-r_{21}^{s, p} r_{23}^{s, p} e^{2 i k_{22} d}\right)$

for the $s$ - and $p$-polarized waves respectively. The corresponding expression (Sipe, 1987) for the Green's function is used when the source and observation points are in different layers.

\section{FLUCTUATION-DISSIPATION THEOREM}

The ensemble average of the currents in Eq. (2) is related to the temperature of the system by the fluctuation-dissipation theorem (Landau et al., 1980) from statistical physics. The theorem relates the microscopic fluctuations of a system in thermodynamic equilibrium to macroscopic, dissipative quantities. For an isotropic and local medium in thermal equilibrium at a temperature $T$, the theorem states that the correlation function of the current density is given by,

$$
\left\langle J_{i}(\mathbf{r}, \omega) J_{j}\left(\mathbf{r}^{\prime}, \omega\right)\right\rangle=\frac{\varepsilon_{o} \varepsilon^{\prime \prime} \omega \Theta(\omega, T)}{\pi} \delta_{i j} \delta\left(\mathbf{r}-\mathbf{r}^{\prime}\right)
$$

where $\varepsilon_{0}$ is the permittivity of free space, $\varepsilon^{\prime \prime}$ is the imaginary part of the dielectric function, $\delta_{i j}$ is the Kronecker delta function, $\delta\left(\mathbf{r}-\mathbf{r}^{\prime}\right)$ is the Dirac delta function, $\Theta(\omega, T)=\hbar \omega /\left[\exp \left(\hbar \omega / k_{B} T\right)-1\right], \hbar$ is Planck's constant divided by $2 \pi$, and $k_{B}$ is Boltzmann's constant. The zero-point energy is neglected in the expression for the average energy of the harmonic oscillator due to reciprocity of the heat transfer conductance. For the net $\hat{x}$-directed flux, substituting Eq. (4) into Eq. (2) gives,

$$
\begin{aligned}
& \left\langle E_{y} H_{z}^{*}-E_{z} H_{y}^{*}\right\rangle= \\
& \frac{i \omega^{2} \mu_{o} \varepsilon_{o} \varepsilon^{\prime \prime} \Theta(\omega, T)}{\pi} \int_{z_{1}}^{z_{2}} d z^{\prime} \int_{-\infty}^{\infty} d y^{\prime} \int_{-\infty}^{0} d x^{\prime}\left(G_{E_{y j}} G_{H_{j z}}^{T^{*}}-G_{E_{z j}} G_{H_{j y}}^{T^{*}}\right)
\end{aligned}
$$

where repeated indices are summed.

\section{NUMERICAL INTEGRATION}

The integration over the $\hat{y}$-direction of the source region in Eq. (5) results in a Dirac delta function which collapses the two integrations over $k_{y}$ into one. However, the corresponding integration in the $\hat{x}$-direction is the Fourier transform of the step function, which consists of two terms given by $\pi \delta\left(k_{x^{\prime}}-k_{x}\right)+i /\left(k_{x^{\prime}}-k_{x}\right)$ where $k_{x}$ is for the $\overrightarrow{\mathbf{G}}_{\mathbf{E}}$ integration and $k_{x}$, is for $\overrightarrow{\mathbf{G}}_{\mathbf{H}}$. The first term leads to the expression for the in-plane flux in the case where the entire film is at the same temperature. As expected, this term does not contribute to the net in-plane flux. Thus the focus is on the second term which introduces a singularity into the integrand whenever $k_{x}$ equals $k_{x^{\prime}}$.

The integral is evaluated numerically using QUADPACK (Piessens, 1983), which is a library of FORTRAN routines for estimating one-dimensional integrals. In particular, a routine using adaptive bisection with Wynn's epsilon algorithm (Wynn, 1956) to speed up the integration of integrable singularities was extended to 4-D to perform the integration over $k_{x}, k_{x}, k_{y}$, and $z^{\prime}$. In addition to the singularity whenever $k_{x}$ equals $k_{x}$, there are also singularities when $z$ equals $z$, when $k_{x}$ and $k_{y}$ simultaneously equal zero, and when $k_{x}$, and $k_{y}$ simultaneously equal zero. The integration is split around each of these singularities as the QUADPACK routine better handles singularities at the endpoints.

The infinite limits of integration in $k_{x}, k_{x}$, and $k_{y}$ lead to a divergence of the flux. This divergence can be avoided by replacing the infinite limit of integration with a finite value, which was chosen to be 


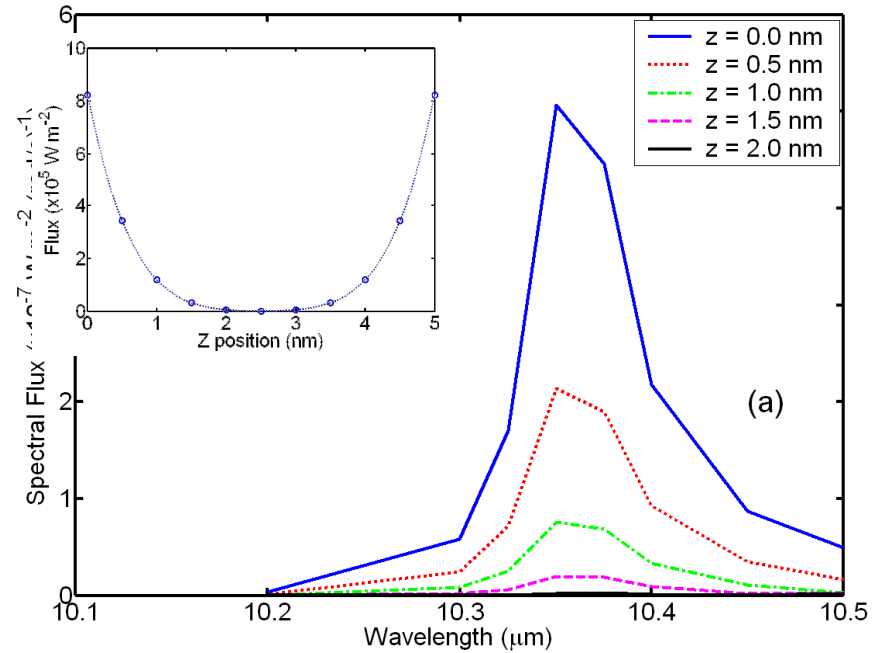

(a)

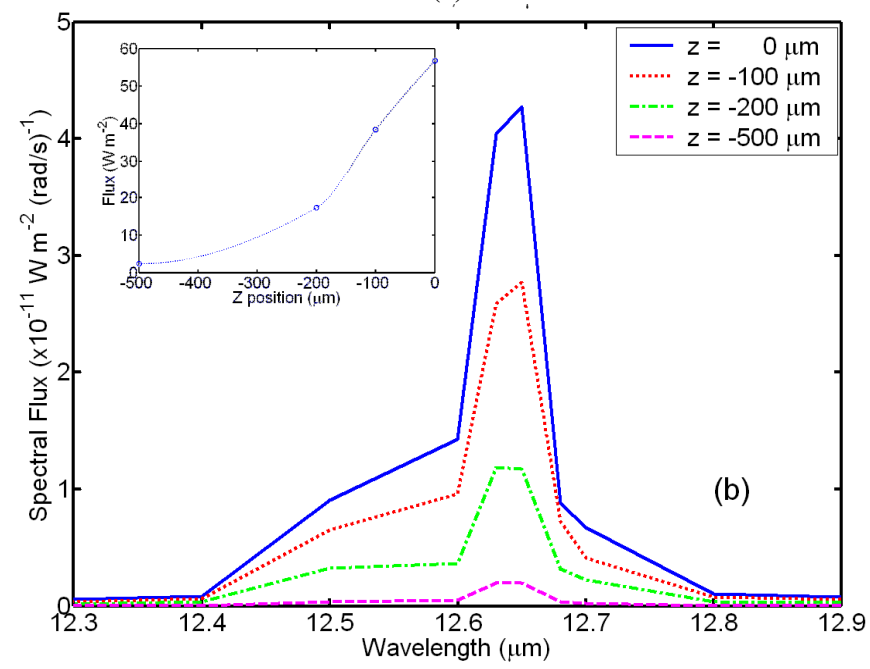

(b)

Fig. 2 (a) A plot of the spectral flux at various $\mathrm{z}$ heights inside a $5 \mathrm{~nm}$ thick silicon carbide film. The flux is calculated $0.5 \mathrm{~nm}$ from the heated edge of the film at $300 \mathrm{~K}$. (b) Plots of the spectral flux at various $\mathrm{z}$ locations outside the film. The respective insets show the spectrally-integrated flux.

$10^{10}$. The effect of this truncation is to set a bound on the smallest $x$ location which can be evaluated. The dependence of the integral on wavevector and position is of the form $\exp \left(-k_{x} x\right)$ so that at a particular $x$ location, the contribution due to increasingly larger wavevectors becomes negligible. Using a criterion of $e^{-5}$, the smallest $x$ position that can be evaluated is $0.5 \mathrm{~nm}$. Due to the oscillatory nature of the integrand, convergence was slow to obtain. With a relative error specification of $10^{-3}$, each spatial location and frequency took between 3 and 72 hours to compute on a $3 \mathrm{GHz}$ Unix workstation with $4 \mathrm{~GB}$ of RAM.

\section{RESULTS AND DISCUSSION}

Calculations were performed for silicon carbide, using a Lorentz model for the dielectric function $\left(\varepsilon_{\infty}=6.7, \omega_{L O}=1.82 \times 10^{14} \mathrm{rad} / \mathrm{s}\right.$, $\omega_{T O}=1.49 \times 10^{14} \mathrm{rad} / \mathrm{s}, \gamma=8.92 \times 10^{11} \mathrm{rad} / \mathrm{s}$ ) which was obtained by a fit to literature values (Palik, 1985). Given the form of the Green's function, it was straightforward to separate the contributions due to the

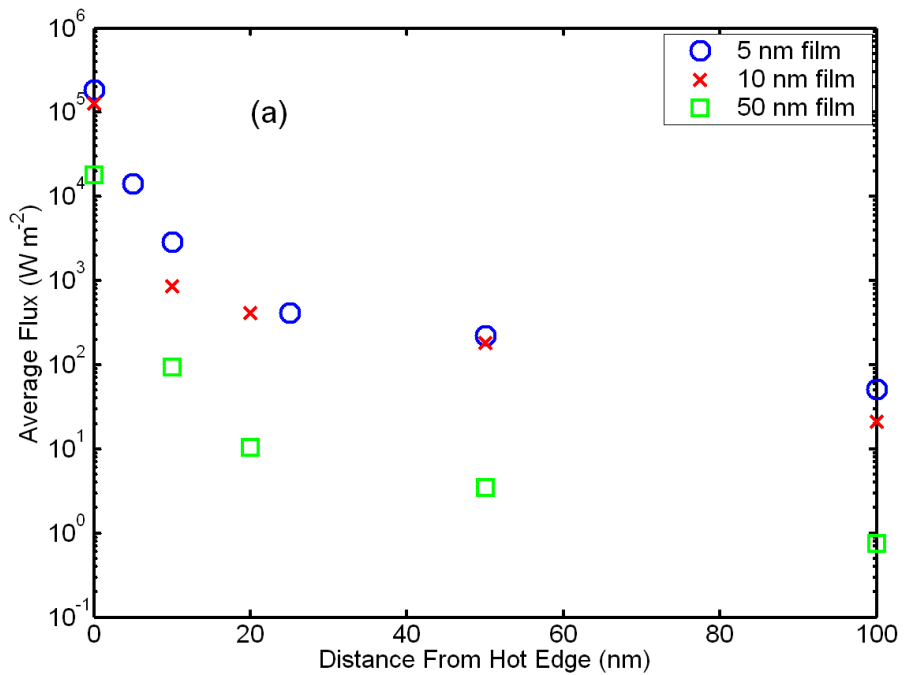

(a)

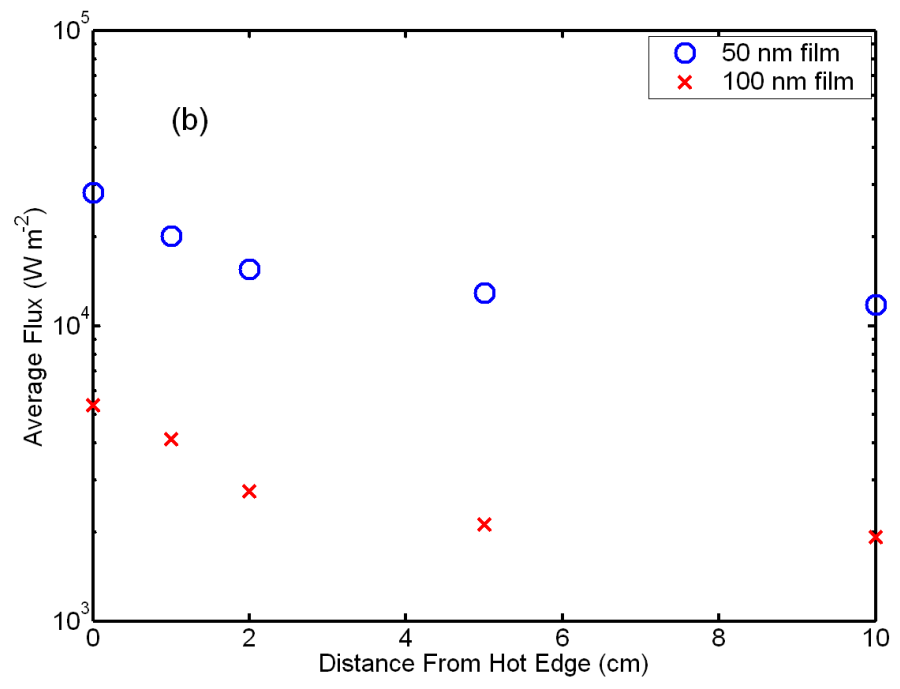

(b)

Fig. 3 The average in-plane flux (a) inside and (b) outside the film as a function of distance from the heated edge for different film thicknesses at $300 \mathrm{~K}$.

transverse electric and the transverse magnetic (TM) modes. As expected, it was found that only the TM mode makes a significant contribution to the flux, which is consistent with the involvement of surface phonon-polaritons.

The spectral flux inside a $5 \mathrm{~nm}$ thick film is plotted in Fig. 2(a) for various $z$ heights in the film. As discussed above, these calculations were done $0.5 \mathrm{~nm}$ from the heated edge of the film, and the inset to the figure shows the spectrally-integrated flux as a function of $z$ location in the film. Figure 2(b) shows the analogous plots for outside the film. It is seen that inside the film, the majority of the in-plane power is carried near the edges of the film, and that it then decays to zero at the center. While the flux outside the film is nearly four orders of magnitude smaller, the penetration into the air is significantly greater, on the order of $100 \mu \mathrm{m}$. At the frequency of the peak spectral flux outside the film, the surface polariton dispersion relation is very close to the light line, indicating that the perpendicular component of the wavevector is very small. As a result, the corresponding penetration depth is quite large.

This trend can be understood by considering that a film will have two surface polaritons, one on each surface. When the film is thin enough, it is well-known that this degeneracy lifts by splitting into an 

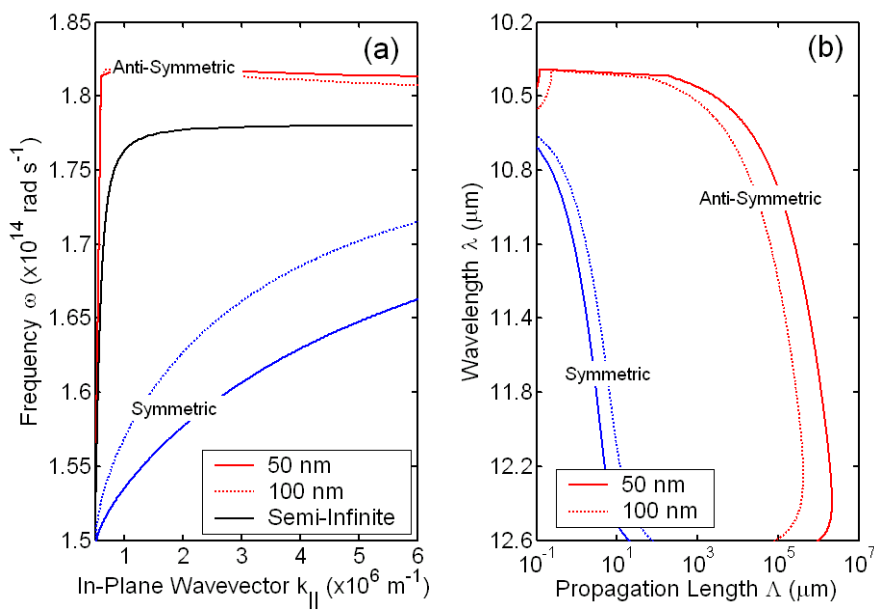

Fig. 4 (a) The surface phonon-polariton dispersion relations for a thin film of silicon carbide. (b) The corresponding propagation lengths.

anti-symmetric and a symmetric mode (Raether, 1988; Sarid, 1982; Economou, 1969). The field of the symmetric mode is mainly in the film, where the damping of the material provides absorption. In contrast, most of the anti-symmetric mode propagates outside the film and penetrates only slightly into the film. With vacuum surrounding the film, the anti-symmetric mode experiences very little damping. Thus, the majority of the in-plane flux propagates outside the film as expected.

Commensurately, the propagation lengths inside and outside the film are very different. After a spline interpolation, the flux is integrated over $z$ and then divided by the film thickness to calculate an average flux at each $x$ location. Plotted in Fig. 3(a) is the average flux inside the film as a function of distance from the heated edge. It is seen that the flux decays exponentially with distance as the energy is absorbed by the film. The propagation lengths inside the film are calculated to be on the order of several nanometers. Outside the film, the average in-plane flux is much higher and has much longer propagation lengths, on the order of tens of centimeters as shown in Fig. 3(b).

The difference in propagation lengths and peak spectral frequencies is explained by considering the thin film dispersion relations (Raether, 1988). Assuming a real frequency, the resulting inplane wavevector is complex (Fukui et al., 1979) where the imaginary part is associated with the propagation length, $\Lambda=1 / 2 \operatorname{Im}\left\{k_{\|}\right\}$, as is done for superlattices (Simkin and Mahan, 2000) and electron energy bands (Pendry, 1974). Inside the film, the peak in the spectral flux corresponds to the high density of states around $10.4 \mu \mathrm{m}$ as indicated by the dispersion relation in Fig. 4(a). The corresponding propagation lengths at this wavelength are less than a micron as shown in Fig. 4(b).Outside the film, the maximum in the spectral flux corresponds with the peak in the imaginary part of the dielectric function of silicon carbide (Palik, 1985) at $12.5 \mu \mathrm{m}$. Although the material damping is large at this frequency, the anti-symmetric mode is mainly outside the film. The dispersion relation shows that at this wavelength the group velocity of the mode is quite large, which results in a long propagation length. The calculated propagation length in Fig. 4(b) is on the order of tens of centimeters, in good agreement with the values extrapolated from the decay in average flux.

The overall in-plane flux as a function of film thickness from the fluctuational electrodynamics calculation compares favorably with previous calculations based on kinetic theory (Chen et al., 2005) as shown in Fig. 5. Interestingly, in both approaches it is seen that thinner films actually transport more flux. This is due to the fact that the antisymmetric mode of the thin film dispersion relation has a propagation length that increases as the film thickness decreases. As the film

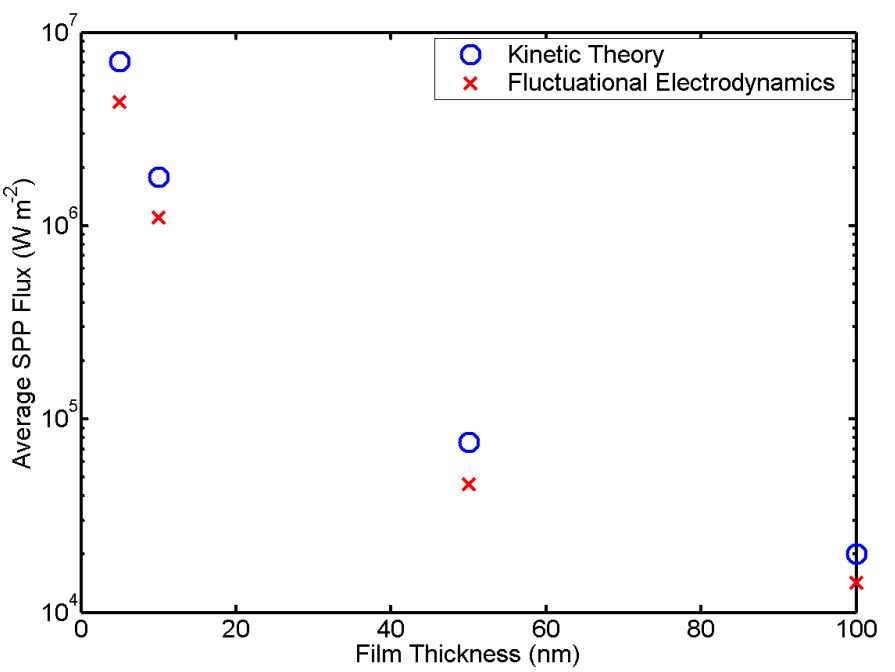

Fig. 5 Comparison of the total in-plane flux from the fluctuational electrodynamics calculation with the kinetic theory results.

thickness increases beyond a critical point given by $k_{z 2} d \approx 1$ (Burke et al., 1986; Raether, 1988; Sarid, 1982; Economou, 1969), there is no longer a splitting of the modes and the effect diminishes.

The phonon and surface phonon-polariton contributions to the thermal conductivity follow distinctly different trends. In general, the phonon contribution decreases as the film thickness decreases because of the increasing effects of surface scattering. In contrast, the thermal conductivity contribution from the surface phonon-polaritons increases with decreasing film thickness as shown in Fig. 5. As discussed in a previous article (Chen et al., 2005), for thin enough films, the surface phonon-polariton contribution to the overall thermal conductivity has the potential to recover the thermal conductivity lost due to these classical size effects.

\section{CONCLUSIONS}

To conclude, we have presented a fluctuational electrodynamics calculation of the in-plane heat flux transported by surface phononpolaritons in nanoscale thin films. As the film thickness decreases, the overall heat flux increases due to the increased propagation length of the anti-symmetric surface phonon-polariton mode. This behavior demonstrates the crucial role of surface polaritons in the transport of energy along thin films. As shown previously (Chen et al., 2005), theeffect is not restricted to crystalline materials, but is also present in amorphous materials, as long as there is a region of negative dielectric function which supports surface polaritons. Although the majority of the in-plane flux is transported in the fields outside the film, it may be possible to design appropriate multi-layer geometries or cladding layers of photonic crystals. If so, this phenomenon suggests a novel method to increase the heat flux along thin films and shows promise in various prospective applications in fields such as microelectronics and optoelectronics.

\section{ACKNOWLEDGEMENTS}

The authors would like to thank Arvind Narayanaswamy for many helpful discussions and Gregg Radtke for guidance regarding the numerical integration. This work is supported by the DOE (Contract No. DE-FG02-02ER45977). 


\section{NOMENCLATURE}

$\begin{array}{ll}c & \text { speed of light in vacuum }(\mathrm{m} / \mathrm{s}) \\ \mathrm{d} & \text { film thickness }(\mathrm{m}) \\ \mathrm{E} & \text { electric field vector }\left(\mathrm{V} \mathrm{m}^{-1}\right) \\ \overrightarrow{\mathbf{G}} & \text { dyadic Green function }\left(\mathrm{m}^{-1}\right) \\ \mathrm{H} & \text { magnetic field vector }\left(\mathrm{A} \mathrm{m}^{-1}\right) \\ \hbar & \text { Planck's constant over } 2 \pi(\mathrm{J} \mathrm{s}) \\ \mathrm{J} & \text { current density }\left(\mathrm{A} \mathrm{m}^{-2}\right) \\ k & \text { wavevector }\left(\mathrm{m}^{-1}\right) \\ k_{B} & \text { Boltzmann's constant }(\mathrm{J} / \mathrm{K}) \\ \mathbf{r} & \text { location vector } \\ T & \text { temperature }(\mathrm{K}) \\ \text { Greek Symbols } \\ \Lambda & \text { propagation length }(\mathrm{m}) \\ \varepsilon & \text { dielectric function } \\ \varepsilon^{\prime \prime} & \text { imaginary part of dielectric function } \\ \mu_{0} & \text { permeability of free space }\left(\mathrm{H} \mathrm{m} \mathrm{m}^{-1}\right) \\ \gamma & \text { damping factor }(\mathrm{rad} / \mathrm{s}) \\ \Theta & \text { mean energy of an oscillator }(\mathrm{J}) \\ \omega & \text { angular frequency }(\mathrm{rad} / \mathrm{s}) \\ \text { Superscripts } & \\ p & \text { p polarization } \\ s & \text { s polarization } \\ \mathrm{T} & \text { matrix transpose } \\ * & \text { complex conjugate } \\ \text { Subscripts } & \\ \text { LO } & \text { longitudinal optical phonon } \\ \text { TO } & \text { transverse optical phonon } \\ 0 & \text { vacuum } \\ 2 & \text { medium }\end{array}$

\section{REFERENCES}

Burke, J.J., Stegeman G.I., and Tamir, T., 1986, "Surface-polariton-like waves guided by thin, lossy metal films," Physical Review B 33, 51865201.

doi:10.1103/PhysRevB.33.5186

Cahill, D.G., Ford, W.K., Goodson, K.E., Mahan, D.G., Majumdar, A., Maris, H.J, Merlin, R., and Phillpot, S.R, 2003, "Nanoscale thermal transport," Journal of Applied Physics, 93, 793-818.

doi:10.1063/1.1524305

Carminati, R. and Greffet, J.-J., 1999, "Near-Field Effects in Spatial Coherence of Thermal Sources," Physical Review Letters 82, 16601663.

doi:10.1103/PhysRevLett.82.1660

Chen, D.-Z. A., Narayanaswamy, A., and Chen, G., 2005, "Surface Phonon-Polariton Mediated Thermal Conductivity Enhancement of Amorphous Thin Films," Physical Review B 72, 155435.

doi:10.1103/PhysRevB.72.155435

Chen, G., 2001 "Phonon Heat Conduction in Low-Dimensional Structures," Semiconductors and Semimetals, Recent Trends in Thermoelectric Materials Research, edited by T. Tritt, Academic Press, San Diego, Vol. 71, pp. 203-259.

Economou, E.N., 1969, "Surface Plasmons in Thin Films," Physical Review 182, 539-554.

doi:10.1103/PhysRev.182.539
Fukui, M., So, V.C.Y., and Normandin, R., 1979, "Lifetimes of Surface Plasmons in Thin Films," Physica Status Solidi (b) 91, K61. doi:10.1002/pssb.2220910159

Goodson, K.E., 1996, “Thermal Conduction in Nonhomogeneous CVD Diamond Layers in Electronic Microstructures," Journal of Heat Transfer, 118, 279-286.

doi: $10.1115 / 1.2825842$

Joulain, K., Mulet, J.-P., Marquier, F., Carminati, R., and Greffet, J.-J., 2005, "Surface Electromagnetic Waves Thermally Excited: Radiative Heat Transfer, Coherence Properties and Casimir Forces Revisited in the Near Field," Surface Science Reports 57, 59-112.

doi:10.1016/j.surfrep.2004.12.002

Landau, L.D., Lifshitz, E.M., and Pitaevskii, L.P., 1980, Statistical Physics, $3^{\text {rd }}$ ed., Pergamon Press, Oxford.

Mills, D.L., 1975, "Attenuation of Surface Polaritons by Surface Roughness," Physical Review B 12, 4036-4046.

doi:10.1103/PhysRevB.12.4036

Mulet, J.-P., Joulain, K., Carminati, R., and Greffet, J.-J., 2001, "Nanoscale Radiative Heat Transfer Between a Small Particle and a Plane Surface," Applied Physics Letters 78, 2931-2933. doi:10.1063/1.1370118

Narayanaswamy, A., and Chen, G., 2003, "Surface Modes for Near Field Thermophotovoltaics," , Applied Physics Letters 82, 3544-3546. doi:10.1063/1.1575936

Narayanaswamy, A. and Chen, G., 2005, "Direct Computation of Thermal Emission from Nanostructures," Annual Review of Heat Transfer, Vol. 14, pp. 169-196.

Palik, E.D., 1985, Handbook of Optical Constants of Solids, Academic Press, Orlando.

Pendry, J.B., 1974, Low Energy Electron Diffraction, Academic Press, New York.

Piessens, R., de Doncker-Kapenga, E., Uberhuber, C.W., and Kahaner, D.K., 1983, QUADPACK A subroutine package for automatic integration, Springer Verlag.

Raether, H., 1988, Surface Plasmons on Smooth and Rough Surfaces and on Gratings, Springer-Verlag, Berlin.

Rytov, S., Kravtsov, Y., and Tatarskii, V., 1989, Principles of Statistical Radiophysics, Springer-Verlag, Berlin.

Sarid, D., 1982 "Long-Range Surface-Plasma Waves on Very Thin Metal Films," Physical Review Letters 47, 1927-1930.

doi:10.1103/PhysRevLett.47.1927

Schoenwald, J., Burstein, E., and Elson, J.M., 1973, "Propagation of Surface Polaritons Over Macroscopic Distances at Optical Frequencies," Solid State Communications 12, 185-189. doi:10.1016/0038-1098(73)90497-3

Shen, S., Narayanaswamy, A., and Chen, G..,"Surface Phonon Polaritons Mediated Energy Transfer between Nanoscale Gaps," Nano Letters, 9, 2909. doi:10.1021/n1901208v 
Simkin, M.V., and Mahan, G.D., 2000, "Minimum Thermal Conductivity of Superlattices," Physical Review Letters 84, 927-930. doi:10.1103/PhysRevLett.84.927

Sipe, J.E., 1987, "New Green-Function Formalism for Surface Optics," Journal of the Optical Society of America B 4, 481-489. doi:10.1364/JOSAB.4.000481
Tsang, L., Kong, J.A., and Ding, K.H., 2000, Scattering of Electromagnetic Waves, Wiley, New York. doi: $10.1002 / 0471224286$

Wynn, P., 1956, "On a Device for Computing the em(Sn) Transformation,” Math. Comp. 10, 91-96. 